\title{
Cross-sectional survey about the prevalence of allergic rhinitis in Argentina: Study PARA
}

\section{Encuesta transversal sobre la prevalencia de rinitis alérgica en Argentina: el estudio PARA}

\author{
Daniel Vázquez, ${ }^{1}$ Iris Medina, ${ }^{1}$ Georgina Logusso, ${ }^{3}$ Sergio Arias, ${ }^{2}$ Gabriel Gattolin, ${ }^{1}$ Claudio Parisi ${ }^{1}$
}

\begin{abstract}
Background: There are very few studies about the prevalence of allergic rhinitis in Argentina.

Objective: To determine the prevalence of allergic rhinitis in a population of inhabitants between the ages of 5 and 44 in Argentina.

Methods: A cross-sectional national study in which a self-reported questionnaire was used. The included participants are between the ages of 5 and 44 and they reside in urban areas. The information was collected by phone.

Results: 3200 participants were surveyed: $51.8 \%$ were women, $37.6 \%$ were between the ages of 5 and 19 , and $62.4 \%$, were between the ages of 20 and 44 . The global prevalence of symptoms of allergic rhinitis was of $20.5 \%$; the most frequent symptoms were sneezing $(58.5 \%)$ and nasal congestion (51.4\%). Overall, $44.3 \%$ of the participants had a family history of allergies. Allergic rhinitis was more frequent in women; the prevalence was of $22.3 \%$ in the group of participants between the ages of 5 and 19 , and of $19.4 \%$ in the group of participants between the ages of 20 and 40 ( $p=0.0545) ; 63.8 \%$ of participants with symptoms did not have a medical diagnosis.

Conclusions: The results of this first cross-sectional national survey have confirmed the high prevalence of self-reported symptoms of allergic rhinitis in adults and children in Argentina, particularly in women.
\end{abstract}

Keywords: Allergic rhinitis; Self-reported questionnaire; Epidemiology; Children; Adults

Este artículo debe citarse como: Vázquez D, Medina I, Logusso G, Arias S, Gattolin G, Parisi C. Encuesta transversal sobre la prevalencia de rinitis alérgica en Argentina: el estudio PARA. Rev Alerg Mex. 2019;66(1):55-64

ORCID

Daniel Vázquez, 0000-0001-9864-0783; Iris Medina, 0000-0001-9559-6756; Georgina Logusso, 0000-0003-0336-6983; Sergio Arias, 0000-0002-1178-4299; Gabriel Gattolin, 0000-0002-8060-516X;

Claudio Parisi, 0000-0002-6936-5599

${ }^{1}$ Asociación Argentina de Alergia e Inmunología Clínica, Buenos Aires, Argentina

${ }^{2}$ Instituto Nacional de Enfermedades Respiratorias, Santa Fe, Argentina

${ }^{3}$ Sanofi Consumer Health Care Cono Sur, Buenos Aires, Argentina
Correspondencia: Daniel Vázquez. drdovazquez@gmail.com

Recibido: 2018-09-04

Aceptado: 2019-01-08

DOI: $10.29262 /$ ram.v66i1.543 


\section{Resumen}

Antecedentes: Existen pocos estudios sobre la prevalencia de rinitis alérgica en Argentina.

Objetivo: Determinar la prevalencia de rinitis alérgica en una población entre cinco y 44 años de edad en Argentina.

Métodos: Estudio transversal nacional en el que se utilizó un cuestionario autoinformado. Se incluyeron participantes entre cinco y 44 años de edad residentes en áreas urbanas. La información se recolectó telefónicamente.

Resultados: Se encuestaron 3200 participantes: $51.8 \%$ del sexo femenino, $37.6 \%$ tenía entre cinco y 19 años y $62.4 \%$ entre 20 y 44 años. La prevalencia global de síntomas de rinitis alérgica fue de $20.5 \%$; los síntomas más frecuentes fueron los estornudos (58.5\%) y la congestión nasal (51.4\%). En general, $44.3 \%$ de los participantes tenía antecedentes familiares de alergia. La rinitis alérgica fue más frecuente en las mujeres; la prevalencia fue de $22.3 \%$ en el grupo de cinco a 19 años y de $19.4 \%$ en el de 20 a 44 años ( $p=0.0545) ; 63.8 \%$ de los participantes con síntomas no tenía diagnóstico médico.

Conclusiones: Los resultados de esta primera encuesta transversal nacional confirman la alta prevalencia de síntomas autoinformados de rinitis alérgica en adultos y niños en Argentina, especialmente en las mujeres.

Palabras clave: Rinitis alérgica; Cuestionario autoinformado; Epidemiología; Niños; Adultos

\author{
Abreviaturas y siglas \\ AN, alergias nasales \\ NPV, valor predictivo negativo
}

PAMI, Programa de Atención Médica Integral

PPV, valor predictivo positivo

$\mathrm{RA}$, rinitis alérgica

\section{Antecedentes}

La rinitis alérgica (RA) es la enfermedad respiratoria crónica más frecuente en todo el mundo, ${ }^{1,2,3}$ con frecuencia está asociada con otras comorbilidades como sinusitis, otitis media y, especialmente, asma, y provoca un deterioro significativo en la calidad de vida, con peores indicadores que enfermedades como la diabetes mellitus, la artritis reumatoidea o la migraña. ${ }^{4}$

Los datos epidemiológicos sugieren que la prevalencia de rinitis alérgica está aumentando en todo el mundo. ${ }^{5}$ De hecho, varios informes indican una tendencia creciente en la prevalencia de esta enfermedad, especialmente en los países en desarrollo, posiblemente relacionada con la contaminación ambiental, los cambios climáticos, el crecimiento urbano y la adopción de estilos de vida similares a los de los países desarrollados. ${ }^{1,5,6,7}$ Se estimó que la prevalencia de RA autoinformada es aproximadamente de 2 a $25 \%$ en los niños y de 1 a $40 \%$ en los adultos. ${ }^{6}$
La RA es un problema de salud mundial. ${ }^{8}{ }^{8}$ Existen escasos datos epidemiológicos en Argentina que indiquen la magnitud y las características de la enfermedad. El objetivo de esta encuesta era realizar un estudio amplio de la prevalencia de RA en Argentina, evaluando una población de cinco a 44 años y recolectar datos del entorno social y clínico.

\section{Método}

Estudio transversal nacional de prevalencia que se realizó a través de una encuesta telefónica para identificar signos y síntomas compatibles con RA, para evaluar sus características y los cuidados recibidos por una población representativa. El consentimiento informado se expuso y registró en forma oral.

Se inscribieron participantes entre cinco y 44 años, residentes de áreas urbanas de Argentina, específicamente de las poblaciones de las aglomeraciones urbanas de más de 100000 habitantes. 
La muestra incluía a 3200 participantes estratificados por sexo y edad. Para calcular el tamaño de la muestra se estimó una prevalencia de rinitis alérgica de $19.5 \%{ }^{10}$ con una precisión relativa de $80 \%$ (variabilidad absoluta de $4 \%$ ) y un nivel de confianza de $95 \%$. Esta prevalencia se propuso a partir del trabajo de prevalencia de RA en Europa, donde los valores obtenidos para España e Italia fueron de 17 y $21.5 \%$, respectivamente, lo cual en combinación resultó aproximadamente en $19.5 \%$. Se consideró que los valores de estos dos países podrían ser aproximados al de Argentina por etnia y hábitos similares. Así, se estimó un tamaño de la muestra de 1600 participantes por sexo para inferir un resultado confiable de la prevalencia; los grupos se definieron como hombres y mujeres, y las poblaciones se definieron en grupos de cinco a 19 años y 20 a 44 años. Para calcular el tamaño de la muestra se utilizó el programa estadístico EPIDAT versión 3.1. La selección de la muestra se realizó utilizando un método de muestreo aleatorizado, estratificado por sexo y grupo de edad, de acuerdo con el tamaño de la población de las aglomeraciones urbanas.

Fueron excluidos los participantes cuyos números telefónicos no correspondían a viviendas particulares u hogares o con incapacidad para comprender o responder las preguntas.

\section{Métodos e instrumentos para la recolección de los datos}

La información se recolectó utilizando un cuestionario formado por secciones sobre datos sociodemográficos generales y factores de riesgo, específicamente preparado por el grupo de investigación, y una sección específica sobre la RA con un cuestionario validado para el diagnóstico. ${ }^{11}$ El cuestionario de diagnóstico incluye componentes sintomáticos; cada pregunta tiene una puntuación asignada de acuerdo con su relevancia para el diagnóstico. La puntuación asignada oscila de 0 a 1; a los participantes que obtenían una puntuación entre 0.75 y 1 se les consideró con diagnóstico de RA. ${ }^{11}$

Si había más de un posible encuestado en los hogares, se numeraban los residentes de cinco a 44 años de cada hogar contactado para seleccionar aleatoriamente a uno. En los participantes de cinco a 17 años, la encuesta era respondida por el adulto responsable. Los participantes de 18 años y mayores respondieron directamente la encuesta. Se registra- ron todas las llamadas, así como la lista de todos los números telefónicos contactados. También se informó la cantidad de llamadas realizadas, los detalles de los rechazos y las llamadas a personas fuera del rango de edad para la encuesta.

De acuerdo con la presencia de síntomas de RA, los encuestados fueron clasificados en "posible rinitis alérgica" o "sin síntomas compatibles con rinitis alérgica".

\section{Variables}

Las variables evaluadas incluyeron las condiciones socioeconómicas y ambientales de los participantes, así como los síntomas compatibles con la RA y factores de riesgo en caso de enfermedad confirmada o sospechada. La variable primaria de estudio fue "casos de rinitis alérgica". Además, se investigaron covariables sociodemográficas: edad, sexo, aglomeración urbana, cobertura del plan de salud, ocupación, nivel de educación; y variables características para la rinitis alérgica: antecedentes familiares de alergia, episodios de resfriado recurrente, frecuencia de episodios de resfrío, síntomas.

\section{Análisis estadístico}

La base de datos se procesó con SPSS Statistics versión 23. La revisión, el ajuste y el procesamiento y análisis completo de la base de datos se realizó en el Instituto Nacional de Enfermedades Respiratorias "Emilio Coni", Santa Fe, Argentina. Con base en las llamadas totales, diferenciando las rechazadas por los encuestados de las que no correspondían a la población del grupo de edad deseado, se calculó el porcentaje de llamadas telefónicas efectivas. Se realizó un análisis descriptivo de las características de la población encuestada utilizando los porcentajes de cada una respecto a la población total que proporcionó información de las variables categóricas; se obtuvo media y desviación estándar de las variables continuas.

Se calcularon la prevalencia de rinitis alérgica y los intervalos de confianza de $95 \%$ utilizando una puntuación de 0.75 en el cuestionario de diagnóstico validado como punto de corte. Se calculó la prevalencia de la muestra completa por edad y sexo. También se evaluaron las características de diagnóstico y las posibilidades de potencial subdiagnóstico de la enfermedad, utilizando porcentajes e intervalos de confianza de $95 \%$. 


\section{Consideraciones éticas}

El estudio fue evaluado y aprobado por un comité de revisión independiente de ética y seguridad y se recopilaron los procedimientos estandarizados para solicitar la autorización y el consentimiento de los encuestados. Si el encuestado pertenecía al grupo de 13 a 17 años, la encuesta era respondida por un padre o tutor legal, con el asentimiento previo del adolescente. El consentimiento o asentimiento de los encuestados se registró grabando la lectura del formulario diseñado con anterioridad. En todo momento se garantizó la confidencialidad de la información a los encuestados.

\section{Resultados}

Se realizaron 15310 llamadas telefónicas y se logró encuestar una muestra final de 3200 participantes (20.9 \%) (figura 1). Por cada encuesta efectiva se realizaron 4.8 llamadas. Las características resumidas de la población se presentan en el cuadro 1 .

Respecto al nivel de educación, $69.9 \%$ de los encuestados del estudio completó la escuela secundaria o educación superior y $0.1 \%$ no tenía educación formal. Además, $10.3 \%$ aún no había completado la educación secundaria y 15.8 \% no había completado la primaria.

En relación con la cobertura médica, $72.1 \%$ tenía algún tipo de cobertura: $51.8 \%$ plan de salud, $23.9 \%$ obra social prepaga o sindical y 1.5 programa o plan de salud pública; $22.8 \%$ no tenía cobertura.

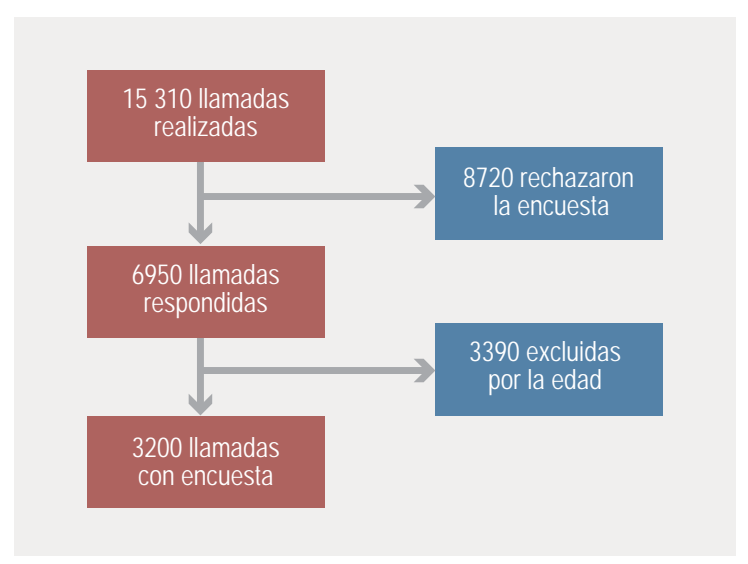

Figura 1. Procedimiento para obtener encuestas efectivas telefónicas para identificar signos y síntomas compatibles con rinitas alérgica.
En 92.1\% de los hogares había un jefe de familia que había trabajado al menos una hora la semana previa a la encuesta. Además, $98 \%$ de los encuestados no vivía en hacinamiento.

\section{Síntomas relacionados con la rinitis alérgica}

Los síntomas más frecuentes entre los encuestados fueron estornudos (58.5\%), congestión nasal (51.4\%) En el cuadro 2 se presenta la distribución proporcional de los síntomas mencionados por los encuestados.

Se observaron resfríos reiterados (congestión nasal, estornudos, picazón en la nariz o secreción nasal) en $41.9 \%$ de los encuestados. Los resfríos se presentaron con mayor frecuencia durante la noche que en la mañana (79.1\%), habitualmente acompañados por síntomas oculares y tos repetida (46.7 y $31.8 \%$ de los encuestados con resfríos, respectivamente). Fue poco común tener obstrucción nasal a causa de olores fuertes $(13.1 \%)$. Respecto a los signos de RA, $51.0 \%$ respondió en forma afirmativa a la presencia de ojeras. Cabe mencionar que $44.3 \%$ de los participantes tenía antecedentes familiares de alergia, como asma, rinitis alérgica, urticaria, alergia a alimentos, a medicamentos, a picaduras de insectos y dermatitis atópica.

\section{Prevalencia de rinitis alérgica}

La prevalencia general de RA obtenida fue de $20.5 \%$. La enfermedad se presentó con mayor frecuencia en las mujeres: $24 \%$ en comparación con $16.7 \%$ de los hombres $(\mathrm{p}=0.0001)$ (cuadro 3$)$.

Se observaron porcentajes diferentes entre los grupos de edad, aunque no estadísticamente significativos, con una prevalencia de RA de $22.3 \%$ entre los sujetos entre cinco y 19 años, y de $19.4 \%$ en los de 20 a 44 años $(p=0.0545)$.

La prevalencia de RA estratificada por sexo y edad se presenta en el cuadro 4; fue menor en el subgrupo de hombres adultos en comparación con los otros subgrupos $(\mathrm{p}<0.0001)$.

\section{Diagnóstico médico de RA}

De los 655 participantes con síntomas consistentes con RA de acuerdo con el cuestionario, 237 fueron diagnosticados por un médico y 418 (63.8\%) no tenían diagnóstico médico. Por otro lado, 221 (48.3\%) de los 458 casos que tenían diagnóstico médico de RA obtuvieron una puntuación que no era compatible con RA según el cuestionario validado. 


\section{Discusión}

Esta encuesta transversal nacional de los síntomas autoinformados de RA confirmó alta prevalencia en Argentina (20.5\%), con predominio en las mujeres. La prevalencia en los hombres adultos es menor que en otros subgrupos. En general, $63.8 \%$ de los participantes con síntomas de RA no tenía diagnóstico médico.

La distribución de la población encuestada por sexo y edad, así como el nivel educativo y la cobertura médica fueron similares a los del último censo nacional de la población. ${ }^{12}$ El índice de respuesta de la encuesta fue similar al de otras encuestas telefónicas. ${ }^{13}$

Los síntomas más frecuentes entre los participantes con RA fueron estornudos $(58.5 \%) \mathrm{y}$ congestión nasal $(51.4 \%)$. Se observaron antecedentes familiares de alergia en $44.3 \%$ de los participantes, similar a lo encontrado en otras encuestas. ${ }^{10,11,14,15,16}$

Se observó gran variabilidad en la prevalencia de RA entre los múltiples estudios, pero en la mayoría la prevalencia fue superior a $15 \%$ y alcanzó hasta $35 \%$, o más en algunos. ${ }^{10,16,17,18,19,20,21}$ Esto indica que la RA es una de las enfermedades respiratorias más comunes, lo que en nuestro estudio es respaldado por la alta prevalencia en la población en general y al dividir a la población en función del sexo y la edad. Se observó mayor prevalencia en las mujeres, hecho que se correlaciona con otros estudios similares; no hubo diferencias significativas al dividir a la población por edades, si bien en algunos análisis se describe mayor frecuencia de RA en la población más joven. ${ }^{19,20,21}$

En Argentina, la prevalencia de RA fue similar a la de algunos países europeos. Bauchau et al. realizaron una encuesta telefónica en más de 9600 participantes $>18$ años en Europa; observaron una prevalencia global de $20.5 \%$, sin embargo, hubo diferencias entre los países: $28.5 \%$ en Bélgica, $24.5 \%$ en Francia, $20.6 \%$ en Alemania, $16.9 \%$ en Italia, $21.5 \%$ en España y $26 \%$ en Reino Unido. ${ }^{10}$ La Encuesta de Salud Respiratoria de la Comunidad Europea identificó una prevalencia similar $(21 \%)$ en 137000 participantes de 20 a 44 años en 22 países europeos. ${ }^{16}$ En ambas investigaciones, la prevalencia fue más alta en los países del norte (Reino Unido y Bélgica) que en los del sur (España e Italia). Se observaron tasas de prevalencia similares en estudios realizados en los Estados Unidos, que consideraban la clasificación de la RA en función de los síntomas

\begin{tabular}{|c|c|c|}
\hline \multicolumn{3}{|c|}{$\begin{array}{l}\text { Cuadro 1. Características demográficas de } 3200 \text { participantes de la } \\
\text { encuesta telefónica en Argentina para identificar signos y síntomas } \\
\text { compatibles con rinitis alérgica }\end{array}$} \\
\hline & $\mathrm{n}$ & $\%$ \\
\hline \multicolumn{3}{|l|}{ Edad (años) } \\
\hline $5-19$ & 1203 & 37.6 \\
\hline $20-44$ & 1997 & 62.4 \\
\hline \multicolumn{3}{|l|}{ Sexo } \\
\hline Mujeres & 1657 & 51.8 \\
\hline Hombres & 1543 & 48.2 \\
\hline \multicolumn{3}{|l|}{ Educación } \\
\hline No escolarizado & 3 & 0.1 \\
\hline Primaria incompleta & 506 & 15.8 \\
\hline Primaria completa & 126 & 3.9 \\
\hline Secundaria incompleta & 331 & 10.3 \\
\hline Secundaria completa & 802 & 25.1 \\
\hline $\begin{array}{l}\text { Educación superior } \\
\text { incompleta }\end{array}$ & 733 & 22.9 \\
\hline Educación superior completa & 638 & 19.9 \\
\hline Posgrado o superior & 61 & 1.9 \\
\hline \multicolumn{3}{|l|}{ Hacinamiento } \\
\hline Tres personas o más & 62 & 2 \\
\hline Menos de tres & 3004 & 94 \\
\hline No informada & 134 & 4 \\
\hline \multicolumn{3}{|l|}{ Ocupación } \\
\hline Sí & 2946 & 92.1 \\
\hline No & 254 & 7.9 \\
\hline \multicolumn{3}{|l|}{ Obra social } \\
\hline Plan de salud PAMI & 1647 & 51.8 \\
\hline Sindical o prepaga & 760 & 23.9 \\
\hline Salud pública & 47 & 1.5 \\
\hline Ninguna & 724 & 22.8 \\
\hline No informada & 22 & 0.7 \\
\hline
\end{tabular}

informados durante más de 30 días y donde las tasas de RA fueron de aproximadamente $20.7 \% .^{19}$

Respecto de los países de América Latina, con el mismo cuestionario validado de nuestro estudio, Mancilla Hernández et al. evaluaron a 8159 participantes de cuatro ciudades de México. Observaron una prevalencia general de RA entre 7 y $22 \%$ (me- 
Cuadro 2. Presentación clínica de la rinitis alérgica identificada en encuesta telefónica

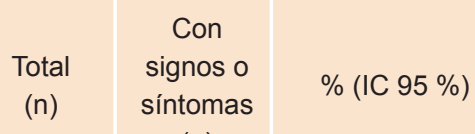

¿Tiene antecedentes familiares de alergia (asma, rinitis alérgica, urticaria, alergia a alimentos, a medicamentos, a picaduras de insectos, dermatitis atópica)?

¿Tiene resfríos repetidos que incluyen congestión nasal, estornudos, picazón en la nariz o secreción nasal?

(n)

¿Con qué frecuencia? (resfríos repetidos)

\begin{tabular}{|c|c|c|c|}
\hline Habitualmente & 1342 & 381 & $28.4(26-30.8)$ \\
\hline Mensualmente & 1342 & 228 & $17(15-19)$ \\
\hline Dos veces por mes & 1342 & 134 & $10(8.4-11.6)$ \\
\hline Cada 2-3 meses & 1342 & 364 & $27.1(24.7-29.5)$ \\
\hline Cada seis meses & 1342 & 149 & $11.1(9.4-12.8)$ \\
\hline Anualmente & 1342 & 76 & $5.7(4.4-6.9)$ \\
\hline \multicolumn{4}{|l|}{ Tiene alguno de los siguientes síntomas? } \\
\hline Congestión nasal & 3200 & 1645 & $51.4(49.7)$ \\
\hline Goteo o hinchazón & 3200 & 1228 & $38.4(36.7-40.1)$ \\
\hline Estornudos & 3200 & 1873 & $58.5(56.8-60.2)$ \\
\hline Picazón de la nariz & 3200 & 1305 & $40.8(39.1-42.5)$ \\
\hline Ningún síntoma & 3200 & 955 & $29.8(28.3-31.4)$ \\
\hline $\begin{array}{l}\text { Di tiene algún síntoma, ¿son más comunes en la noche o mañana, } \\
\text { en comparación con el resto del día? }\end{array}$ & 2245 & 1776 & $79.1(77.4-80.8)$ \\
\hline Si tiene algún síntoma de resfrío, ¿está acompañado por tos repetida? & 2245 & 715 & $31.8(29.9-33.8)$ \\
\hline \multicolumn{4}{|l|}{ Con qué frecuencia? (si los síntomas son acompañados por tos repetida) } \\
\hline Siempre & 715 & 75 & $10.5(8.2-12.7)$ \\
\hline Casi siempre & 715 & 181 & $25.3(22.1-28.5)$ \\
\hline A veces & 715 & 459 & $64.2(60.7-67.7)$ \\
\hline Tiene estornudos consecutivos (más de tres estornudos consecutivos)? & 3200 & 1270 & $39.7(38-41.4)$ \\
\hline El resfrío le tapa la nariz (nariz tapada)? & 3200 & 1502 & $46.9(45.2-48.7)$ \\
\hline Los olores fuertes le tapan la nariz? & 3200 & 419 & $13.1(11.9-14.3)$ \\
\hline Tiene picazón en la nariz? & 3200 & 1273 & $39.8(38.1-41.5)$ \\
\hline $\begin{array}{l}\text { os síntomas nasales, ¿son acompañados por s } \\
\text { lagrimación, picazón en los ojos)? (si tiene algúr }\end{array}$ & 2494 & 1164 & $46.7(44.7-48.6)$ \\
\hline
\end{tabular}

¿Con qué frecuencia los síntomas nasales están acompañados de síntomas oculares?

\begin{tabular}{lrrc} 
Siempre & 1164 & 172 & $14.8(12.7-16.8)$ \\
\hline Casi siempre & 1164 & 292 & $25.1(22.6-27.6)$ \\
\hline A veces & 1164 & 700 & $60.1(57.3-63)$ \\
\hline ¿Tiene una línea o una marca en la nariz? & 3200 & 281 & $8.8(7.8-9.8)$ \\
\hline ¿Tiene ojeras cuando se siente mal? & 3200 & 1633 & $51(49.3-52.8)$
\end{tabular}


dia de $15 \%$ ) en los adolescentes de escuela secundaria e intermedia, y entre 8 y $15 \%$ (media del $13 \%$ ) en los niños de preescolar y primaria. Las tasas fueron más favorables en las adolescentes mujeres y en los niños varones. ${ }^{22}$ Con el cuestionario de la fase III del ISAAC en adolescentes entre 13 y 14 años, Asher et al. informaron una prevalencia de $10.7 \%$ en Brasil, $16 \%$ en Chile, $16.2 \%$ en Paraguay y $34.5 \%$ en Uruguay; ${ }^{15}$ en Argentina fue de $34.9 \% .{ }^{15}$

En otra encuesta telefónica en América Latina, Neffen et al. describieron síntomas, impacto y tratamiento de las alergias nasales (AN) en 1088 adultos y 457 niños $\geq 4$ años. ${ }^{5}$ Se realizó un estudio en Argentina, Brasil, Chile, Colombia, Ecuador, México, Perú y Venezuela y se obtuvo una prevalencia general de RA por diagnóstico médico de $6.6 \%$, pero de $3.5 \%$ en Argentina. ${ }^{5}$ Cabe mencionar que la muestra de Argentina estuvo integrada por 45 niños y 173 adultos..$^{5}$ La congestión nasal fue el síntomas más común y molesto de las AN. Las personas que la sufrían indicaron que los síntomas afectaban la productividad y el sueño y tenían un impacto negativo sobre la calidad de vida. ${ }^{5}$ En nuestro estudio, los criterios de selección para las aglomeraciones, la distribución según cada aglomeración y jurisdicción y el procedimiento de cálculo de tal distribución fueron características metodológicas distintivas. Se inscribieron 3200 participantes de 28 aglomeraciones urbanas, por lo tanto, esta es la primera encuesta transversal nacional de los síntomas de RA autoinformados con este tipo de diseño.

Existen distintos factores que podrían explicar las diferencias de la prevalencia entre los estudios europeos y los latinoamericanos. La genética puede ser un motivo, sin embargo, podría haber cuestiones metodológicas que afecten los resultados.

El cuestionario autoinformado es una herramienta muy utilizada en las investigaciones epidemiológicas de un gran número de participantes y para correlacionar con el diagnóstico médico de la RA. ${ }^{10,15,16,22,23}$

Las encuestas telefónicas basadas en cuestionarios validados se han empleado en otras investigaciones. ${ }^{10,11,14,15,16,22}$ La validación del cuestionario para adultos utilizado en esta encuesta mostró los siguientes resultados: $91 \%$ de sensibilidad, $89 \%$ de especificidad, $89 \%$ de valor predictivo positivo (PPV) y $92 \%$ de valor predictivo negativo (NPV), mientras que el cuestionario para niños demostró $92 \%$ de sensibilidad y $93 \%$ de especificidad, $93 \%$

\begin{tabular}{|c|c|c|c|}
\hline & $\mathrm{n}$ & $\%$ & IC $95 \%$ \\
\hline \multicolumn{4}{|l|}{ Sexo } \\
\hline Mujeres & 397 & 24 & 21.9-26.0 \\
\hline Hombres & 258 & 16.7 & 14.9-18.6 \\
\hline \multicolumn{4}{|l|}{ Edad (años) } \\
\hline $5-19$ & 268 & 22.3 & $19.9-24.6$ \\
\hline 20- 44 & 387 & 19.4 & $17.6-21.1$ \\
\hline Total & 655 & 20.5 & $19.1-21.9$ \\
\hline
\end{tabular}

Población total $N=3200$; población con rinitis alérgica $n=655$.

Punto de corte de 0.75 para la puntuación del cuestionario validado en Argentina.

de PPV y $92 \%$ de NPV con un análisis de homogeneidad de 0.7 (alpha de Cronbach). ${ }^{11}$ El cuestionario del ISAAC para la RA se validó en los rangos de seis y siete años y 13 a 14 años. ${ }^{15}$ En este estudio se utilizó otro cuestionario validado para niños y adultos. ${ }^{11}$

Otra diferencia metodológica es que el cuestionario de diagnóstico que utilizaron Mancilla et al. detecta la RA mediante una puntuación final. ${ }^{11,22}$ En el cuestionario del ISAAC, cada pregunta tiene una sensibilidad y especificidad diferente y las preguntas son independientes una de otra. ${ }^{15}$ Distintas encuestas en las que se utilizó el cuestionario del ISAAC determinaron un rango prevalencia de RA amplio, especialmente en las preguntas sobre el diagnóstico médico, probablemente debido al subdiagnóstico de la enfermedad. ${ }^{24}$ Otra consideración

\begin{tabular}{|c|c|c|}
\hline \multicolumn{3}{|c|}{$\begin{array}{l}\text { Cuadro 4. Prevalencia de rinitis alérgica estratificada por sexo y } \\
\text { edad identificada en encuesta telefónica }\end{array}$} \\
\hline & $n$ & $\%($ IC $95 \%)$ \\
\hline \multicolumn{3}{|c|}{ Edad de las mujeres (años) } \\
\hline $5-19$ & 128 & $21.2(17.9-24.4)$ \\
\hline $20-44$ & 269 & $25.6\left(22.9-28.2^{*}\right.$ \\
\hline \multicolumn{3}{|c|}{ Edad de los hombres (años) } \\
\hline $5-19$ & 140 & $23.9(19.5-28.4)$ \\
\hline $20-44$ & 118 & $12.5(10.4-14.6)^{\star *}$ \\
\hline \multicolumn{3}{|c|}{$\begin{array}{l}\text { Población total } N=3200 \text {, población con rinitis alérgica } n=655 \text {. } \\
\text { Punto de corte de } 0,75 \text { para el puntaje del cuestionario validado en } \\
\text { Argentina. } \\
{ }^{*} p=N S,{ }^{* \star} p<0.0001 \text {. }\end{array}$} \\
\hline
\end{tabular}


metodológica es que probablemente los participantes con un diagnóstico previo tengan síntomas más graves que los participantes con menos síntomas en un cuestionario autoinformado como el nuestro y el utilizado en el ISAAC. ${ }^{11,15,22}$ El formulario SFAR, empleado mayormente en Europa, aplica el procedimiento mencionado, aunque las mismas preguntas o claves para el diagnóstico. Otro formulario común es el SNOT 20, que se limita a las personas en quienes la enfermedad ya ha sido diagnosticada más que para determinar la enfermedad. ${ }^{25,26}$ No existen estudios comparativos entre los distintos tipos de cuestionarios, pero esto podría explicar por qué se determinó una prevalencia de RA entre 3.5 y $34.9 \%$ en Argentina. $5,15,22$

Observamos una prevalencia más alta de RA en las mujeres adultas y más baja en los hombres adultos respecto a los otros subgrupos (cuadros 3 y 4). En otras encuestas telefónicas también se detectó predominio de las mujeres. ${ }^{5,10,16}$ En el estudio mexicano solo se observó en los adolescentes, pero no en los niños. ${ }^{22}$ Puede haber un sesgo de selección con impacto en las observaciones. ${ }^{6}$

En el presente estudio solo tenían diagnóstico médico $36.2 \%$ de los casos de RA identificados por el cuestionario. No obstante, la prevalencia de diagnóstico autoinformado y confirmado por el médico fue similar en otras encuestas. . $^{5,10,16}$

Una potencial limitación de nuestro estudio, al igual que otros realizados por encuestas telefónicas, es la posibilidad de sobreestimación de la prevalencia debido a que las personas con síntomas pudieron haber participado con mayor frecuencia. Otro problema de las encuestas telefónicas es la limitación de la representatividad de la muestra en términos de las condiciones socioeconómicas, en relación con la que puede tener la población en general, dado que es plausible considerar que la aquella con condición socioeconómica más baja no se encontrará suficientemente representada por su menor disponibilidad de teléfono fijo. Adicionalmente se debe considerar también la limitación o problemática de la diversidad de instrumentos para la valoración de RA; nosotros seleccionamos uno validado contra diagnóstico médico de RA. Los diferentes instrumentos existentes para valorar la condición compatible con RA no suelen tener comparación y valoración entre sí, lo cual circunscribe la comparación de resultados cuando se hace con mediciones tomadas con otros instrumentos.

En conclusión, las observaciones de esta encuesta transversal nacional en niños y adultos confirman una alta prevalencia de síntomas autoinformados de RA, con predominio en las mujeres, prevalencia menor en hombres adultos e índice de subdiagnóstico médico significativo. En función de estos resultados, en Argentina 5.5 millones de personas entre cinco y 44 años están afectadas por RA. Teniendo en cuenta el elevado número de personas afectadas y el hecho de que la RA tiene un impacto importante sobre la calidad de vida, la mejora y el ajuste de los criterios y el acceso al diagnóstico y tratamiento podrían reducir o mitigar el impacto personal y social de esta enfermedad. Se espera que la información obtenida permita el diseño e implementación de las medidas necesarias para reducir la carga de esta enfermedad respiratoria crónica y sus comorbilidades en la calidad de vida de los pacientes.

\section{Conflicto de intereses}

Georgina Logusso actualmente es empleada de Sanofi-Argentina. El resto de los autores declararon que no tienen conflictos de intereses.

\section{Financiación}

El estudio fue patrocinado por SANOFI-Argentina.

\section{Referencias}

1. Brozek JL, Bousquet J, Baena-Cagnani CE, Bonini S, Canonica GW, Casale TB, et al. Allergic Rhinitis and its Impact on Asthma (ARIA) guidelines: 2010 revision. J Allergy Clin Immunol. 2010;126:466-476. DOI: $10.1016 /$ j.jaci.2010.06.047

2. Hansel FK. Clinical and histopathologic studies of the nose and sinuses in allergy. J Allergy Clin Immunol. 1929;1(1):43-70. DOI: 10.1016/S0021-8707(29)90083-6

3. Linneman L, Mayrga-Butrón JL, Sánchez-González A, Ramírez-García A, Medina-Ávalos M, FigueroaMorales MA, et al. ARIA México 2014. Adaptación de la Guía de Práctica Clínica ARIA 2010 para México. 
Metodología ADAPTE. Rev Alerg Mex. 2014;61(Supl 1):S3-S116. Disponible en: http://revistaalergia. mx/ojs/index.php/ram/article/view/52/94

4. Breinbauer H, Varela C, Núñez M, Ugarte S, Garfias R, Fonseca X. Encuesta de síntomas SNOT20 para rinitis alérgica y rinosinusitis: validación en Chile. Rev Med Chile. 2011;139(7):886-895. DOI: 10.4067/S0034-98872011000700009

5. Neffen H, Mello JF, Sole D, Naspitz CK, Dodero AE, Garza HL, et al. Nasal allergies in the Latin American population: results from the Allergies in Latin America Survey. Allergy Asthma Proc. 2010;31(Suppl 1):S9-S27. DOI: 10.2500/aap.2010.31.3347

6. Brozek JL, Bousquet J, Agache I, Agarwal A, Bachert C, Bosnic-Anticevich S, et al. Allergic Rhinitis and its impact on Asthma (ARIA) guidelines: 2016 revision. J Allergy Clin Immunol. 2017;140(4):950-958. DOI: 10.1016/j.jaci.2017.03.050

7. Wheatley LM, Togias A. Clinical practice. Allergic rhinitis. N Engl J Med. 2015;372(5):456-463. DOI: 10.1056/NEJMcp1412282

8. Neffen HE. How can we improve management of allergic rhinitis in Latin America? Allergy Asthma Proc. 2010;31(Suppl 1):S5-S7. DOI: 10.2500/aap.2010.10.3339

9. Asher MI, Montefort S, Björkstén B, Lai CK, Strachan DP, Weiland SK, et al. Worldwide time trends in the prevalence of symptoms of asthma, allergic rhinoconjuntivitis, and eczema in childhood: ISAAC Phases One and Three repeat multicountry cross-sectional surveys. Lancet. 2006;368(9537):733-743. DOI: 10.1016/S0140-6736(06)69283-0

10. Mancilla-Hernández E, Medina-Ávalos M, Osorio-Escamilla RE. Validación de un cuestionario diagnóstico de rinitis alérgica para estudios epidemiológicos. Rev Alerg Mex. 2014;61:153-161. DOI: 10.29262/ram.v61i3.40

11. Mancilla-Hernández E, Medina-Ávalos M, Barnica-Alvarado RH, Soto-Candia D, Guerrero-Venegas R, Zecua-Nájera Y. Prevalence of rhinitis allergic in populations of several states of Mexico. Rev Alerg Mex. 2015;62(3):196-201. Disponible en: http://revistaalergia.mx/ojs/index.php/ram/article/view/107/208

12. All-Khaled N, Pearce N, Anderson HR, Ellwood P, Montefort S, Shan J, et al. Global map of the prevalence of symptoms of rhinoconjuctivitis in children: the International Study of Asthma and Allergies in Childhood (ISAAC) Phase Three. Allergy. 2009;64(1):123-148. DOI: 10.1111/j.1398-9995.2008.01884.x

13. Katelaris CH, Lee BW, Potter PC, Maspero JF, Cingi C, Lopatin A, et al. Prevalence and diversity of allergic rhinitis in regions of the world beyond Europe and North America. Clin Exp Allergy. 2012;42(2):186-207. DOI: 1111/j.1365-2222.2011.03891.x

14. Bousquet J, van Cauwenberge P, Khaltaev N, Aria Workshop Group; World Health Organization. Allergic rhinitis and its impact on asthma. J Allergy Clin Immunol. 2001;108(Suppl 1):S147:S334.

15. Zuberbier T, Lötvall J, Simoens S, Subramanian SV, Church MK. Economic burden of inadequate management of allergic diseases in the European Union: a GA (2) LEN review. Allergy. 2014;69(10):1275-1279

16. Argentina - National Population, Households, and Dwellings Census 2010. Argentina: National Institute of Statistics and Census; 2017.

17. Vidal Díaz-De Rada, Palacios-Gómez JL. Comparación de las tasas de respuestas en el uso combinado de modalidades de encuestas. Rev Esp Investig Soc. 2013;141:159-170.

18. Bauchau V, Durham SR. Prevalence and rate diagnosis of allergic rhinitis in Europe. Eur Respir J. 2004;24(5):758-764. DOI: 10.1183/09031936.04.00013904

19. Janson C, Anto J, Burney P, Chinn S, De Marco R, Heinrich J, et al. The European Community Respiratory Health Survey: what are the main results so far? European Community Respiratory Health Survey II. Eur Respir J. 2001;18(3):598-611. Disponible en: https://erj.ersjournals.com/content/18/3/598.long

20. Nathan R, Meltzer EO, Derebery J, Campbell UB, Stang PE, Corrao MA, et al. The prevalence of nasal symptoms attributed to allergies in the United States: findings from the burden of rhinitis in an America survey. Allergy Asthma Proc. 2008;29(6):600-608. DOI: 10.2500/aap.2008.29.3179

21. Klossek JM, Annesi-Maesano I, Pribil C, Didier A. Un tiers des adultes ont une rhinite allergique en France (enquête INSTANT). Presse Med. 2009;38(9):1220-1229. DOI: 10.1016/j.lpm.2009.05.012 
22. Björkstén B, Clayton T, Ellwood P, Stewart A, Strachan D, ISAAC Phase Three Study Group. Worldwide time trends for symptoms of rhinitis and conjunctivitis: phase III of the International Study of Asthma and Allergies in Childhood. Pediatr Allergy Immunol. 2008;19(2):110-124. DOI: 10.1111/j.13993038.2007.00601.x

23. Pynnonen MA, Kim HM, Terrell JE. Validation of the Sino-Nasal Outcome Test 20 (SNOT-20) domains in non-surgical patients. Am J Rhinol Allergy. 2009;23(1):40-45. DOI: 10.2500/ajra.2009.23.3259

24. Nogueira-Silva L, Martins SV, Cruz-Correia R, Azevedo LF, Morais-Almeida M, Bugalho-Almeida A, et al. Control of allergic rhinitis and asthma test formal approach to the development of a measuring tool. Respir Res. 2009;10:52. DOI: 10.1186/1465-9921-10-52

25. Kim H, Levin L, LeMasters GK, Villareal M, Evans S, Lockey JE, et al. Validating childhood symptom with physician diagnosed allergic rhinitis. Ann Allergy Asthma Immunol. 2012;108(4):228-231. DOI: 10.1016/j.anai.2012.02.004

26. Del Río-Navarro B, Luna-Pech JA, Berber A, Zepeda-Ortega B, Ávila-Castañón L, Del Río Chivardi $\mathrm{JM}$, et al. Factors associated with allergic rhinitis in children from northern Mexico city. J Investig Clin Immunol. 2007;17(2):77-84. Disponible en: http://www.jiaci.org/issues/vol17issue02/3.pdf 